Témoigner Témoigner. Entre histoire et mémoire

Getuigen Revue pluridisciplinaire de la Fondation Auschwitz

$116 \mid 2013$

Voyages mémoriels

\title{
Au-delà d'une morale de la représentation : un nouveau rapport à la mémoire
}

Beyond the right and wrong of representation; a new relation to memory

Voorbij de moraal en de voorstelling: een nieuwe vorm van herinnering

\section{Gabriel Raichman}

\section{(2) OpenEdition}

Journals

\section{Édition électronique}

URL : http://journals.openedition.org/temoigner/343

DOI : 10.4000/temoigner.343

ISSN : 2506-6390

Éditeur :

Éditions du Centre d'études et de documentation Mémoire d'Auschwitz, Éditions Kimé

Édition imprimée

Date de publication : 1 septembre 2013

Pagination : 84-90

ISBN : 978-2-84174-643-9

ISSN : 2031-4183

Référence électronique

Gabriel Raichman, « Au-delà d'une morale de la représentation : un nouveau rapport à la mémoire »,

Témoigner. Entre histoire et mémoire [En ligne], 116 | 2013, mis en ligne le 01 juin 2015, consulté le 23 octobre 2020. URL : http://journals.openedition.org/temoigner/343 ; DOI : https://doi.org/10.4000/ temoigner.343 


\section{Au-delà d'une morale de la représentation : un nouveau rapport à la mémoire}

GABRIEL RAICHMAN

$I A D$, Louvain-la-Neuve

1 semblerait que l'Amérique d'aujourd'hui ait des comptes à régler avec son passé, c'est en tout cas ce qui apparaît lorsque l'on s'intéresse au dernier film de deux des grands maîtres américains, Spielberg et Tarantino. En effet, tous deux sont à l'heure actuelle des acteurs du discours mémoriel, traitant chacun la question de la Shoah et de la Seconde Guerre mondiale dans leur filmographie antérieure. Dans leur œuvre la plus récente, ils continuent à explorer cette dimension pour se concentrer sur une époque que les Américains ont encore du mal à assumer : celle englobant la guerre de Sécession et la fin de l'esclavage.

Lincoln nous met face aux images de la démocratie (débats à la Chambre des représentants, rencontres secrètes, corruption), livrées selon un mode de lecture à première vue paradoxal : les hommes politiques se font juristes et débattent inlassablement autour du sens de chaque notion pour en donner une définition sur laquelle ils puissent s'accorder (nature, liberté, égalité, justice, etc.). Lincoln lui-même fait sans cesse référence à ses connaissances en droit, aussi bien lorsqu'il s'agit d'appliquer la loi pour résoudre de simples problèmes quotidiens que pour philosopher librement sur les notions ordinaires d'Euclide et en tirer des postulats sur la justice. Spielberg met en œuvre une grande énergie pour nous faire comprendre, dans l'approche didactique qui caractérise parfois son œuvre, comment le processus démocratique repose sur un code et des conventions établies, mais constamment renégociées (à l'image de cette tournure juridique qu'utilise le président pour éviter que ne soit repoussée la date du vote, considérée par les démocrates comme un « détour d'avocat »). La recherche de la légalité est donc le propre de la démocratie.

Mais le régime fonctionne aussi dans l'ombre, à la limite de cette légalité: Lincoln offre ainsi d'importants postes à des représentants démocrates afin qu'ils votent en faveur du $13^{\mathrm{e}}$ Amendement. L'opposition est donc claire entre d'un côté le caractère précis et juste de la démarche démocratique «officielle » et l'obscurité, le flou (la 
frontière entre légalité et illégalité est extrêmement ténue et semble même parfois inexistante) ainsi que la dimension éminemment complexe (il sagit justement de jouer sur ce droit parfois polysémique pour arriver à ses fins) du jeu politique «privé ».

Spielberg s'intéresse finalement à la démonstration d'une intelligence politique hors du commun, qu'il cherche à capter en pleine manifestation. Lincoln est souvent plongé dans sa réflexion lorsque la caméra du réalisateur l'isole à l'intérieur du cadre: sa pensée profonde, ses doutes, ses aspirations, ses craintes ne peuvent être compris par les autres politiciens (conseillers ou représentants), enracinés dans un pragmatisme politique qui les rend incapables de voir l'Idée qui habite le président et guide son action (seul Thaddeus Stevens ${ }^{1}$ sera capable, selon ses propres mots, $\mathrm{d}^{\prime}$ «être étonné »).

Lincoln garde en effet un calme profond durant l'essentiel du film (à quelques exceptions près), son visage grave plongé dans le clair obscur de l'image, tandis quautour de lui règne une agitation de tous les instants. Lui seul est décidé et inflexible, là où les autres sont en proie à une perpétuelle remise en question : des démocrates changeant de bord après que des postes leur ont été offerts, ses conseillers acceptant peu à peu sa décision, les émissaires confédérés reconnaissant l'abolition de l'esclavage. Il est comme un roc autour duquel gravite le monde politique de son époque, les tendances et les opinions qui s'effritent à son contact pour finalement rejoindre sa pensée visionnaire.

De plus, la mise en scène déployée dans Lincoln est foncièrement réaliste. Le cinéaste allie longs plans descriptifs (à l'aide de mouvements de caméra lents et parfaitement fluides permettant petit à petit de découper le champ, d'élargir ou de resserrer l'espace, de se centrer sur des points essentiels, souvent d'ailleurs la figure du président) et grande profondeur de champ, autrement dit deux points considérés comme révélateurs du réalisme cinématographique depuis les travaux d'André Bazin.

Spielberg vise l'immersion du spectateur dans son univers diégétique, premièrement en insistant sur la continuité (aussi bien spatiale que temporelle) du film, mais également par l’objectif sans cesse réaffirmé de réussir une parfaite reconstitution historique.

Le réalisme ne sert cependant pas ici un propos critique ayant pour but d'explorer et d'exposer les failles du système démocratique.

Au contraire, la juxtaposition de plans à la composition picturale et dramatique (du fait notamment des jeux de lumière) enferme le sujet dans un sérieux et une solennité qui permet au cinéaste de donner une grandeur à sa représentation d'Abraham Lincoln, de le changer en homme providentiel, notamment dans la séquence où on le voit sur son lit de mort, seul au milieu de ses disciples, recouvert d'un halo de lumière.

Spielberg réalise ainsi ce qui pourrait s’apparenter à une utopie démocratique : une figure messianique et inflexible capable de faire voter «la plus grande mesure du XIX siècle, passée par corruption, aidée et incitée par l'homme le plus pur d'Amérique.» Cette pureté, celle du martyre, magnifie la démocratie et en rachète les fautes, toutes

(1) Élu de la Pennsylvanie, il a été un des membres les plus influents de la Chambre des représentants et un des leaders de l'aile radicale du parti républicain. 
les manœuvres pouvant être considérées comme moralement discutables acquièrent ainsi leur légitimité et même leur grandeur à l'ombre de l'œuvre de Lincoln.

Nous touchons donc ici au véritable propos du film : celui-ci ne porte aucune représentation de l'esclavage ou de la guerre de Sécession (bien que les quelques images de la guerre nous rappellent, lors de scènes aussi brèves que puissantes - visite du charnier, affrontement ouvrant le film - la réalité matérielle à côté de l'intelligence et de la réflexion politique) et se contente de montrer le politique à l'œuvre. Les images, réalistes, se voudraient presque documentaires ${ }^{2}$ s'il n'y avait la figure du président pour leur donner un caractère onirique, les transformer en souvenir d'une démocratie idéaliste, les ancrer dans nos mémoires comme un possible.

Tarantino, dans son film Django Unchained, place au contraire le public au cœur de cette souffrance que Spielberg laisse de côté. L'image du pouvoir est quasiment absente tout au long du récit, le spectateur se trouve plongé au sein d'un monde qui ne repose sur aucune loi. Il ne s'agit plus ici d'un discours sur la démocratie, sur ses institutions et son organisation, mais de l'envers du décor, un récit de la violence quotidienne, de la souffrance liée à l'esclavage, un récit sur la barbarie des hommes, loin de l'idéalisme et de l'espoir que symbolisait la figure de Lincoln.

Malgré cet aspect trivial, l'esthétique du film est opposée à un quelconque réalisme: Tarantino ne vise pas la reconstitution historique, il affirme à chaque instant sa place de réalisateur et son souci constant de mise en scène. Une distance se crée entre son sujet et le spectateur, contrairement à ce qui se produit dans le film de Spielberg qui recherchait l'immersion : cette distance lui permet de ne plus se préoccuper des exigences de vraisemblance et donc de réaliser sa propre représentation de l'esclavage. Pour mieux comprendre cette question, il devient nécessaire de se pencher brièvement sur la filmographie du réalisateur.

Cinéaste de la démesure depuis toujours, celle-ci passait notamment, dans ses premiers films (Reservoir Dogs et Pulp Fiction en tête), par un dispositif narratif complexe, qu'il est d'ailleurs l'un des premiers à avoir exploité de la sorte et qui fut repris par la suite pour être appliqué mécaniquement dans beaucoup de grandes productions hollywoodiennes : cela ne veut pas dire cependant que tous les films ayant repris ces méthodes soient condamnés à la médiocrité, mais simplement que Tarantino est le premier à avoir osé bouleverser totalement le rapport réaliste et linéaire au temps ; une fois le spectateur habitué à cette possibilité, le parti pris semble nettement moins audacieux, et pourrait presque aujourd'hui relever d'un schéma classique.

Ses premiers films étaient comme des univers clos, le réalisateur y développait les mêmes figures, des situations semblables, une esthétique « pop » stylisée (davantage dans Pulp Fiction que Reservoir Dogs, au style plus épuré). Aucun dialogue n’y existait véritablement, si ce n'est celui de l'oeuvre avec elle-même. Ces obsessions, qui

(2) L'aspect documentaire favorise l'immersion du spectateur dans l'univers fictionnel, l'aide à s'ancrer dans la réalité qui lui est montrée. Spielberg cherche donc à la fois à établir une forme de réalisme immersif tout en reniant la dimension événementielle de son sujet, la figure de Lincoln transcendant le cadre du récit pour lui donner une dimension intemporelle et utopique. 
revenaient fréquemment (avec toutefois des variations), ces délires, ont entretenu une mythologie qui valut au cinéaste le succès qu'on lui connaît et la force de son style. Entrer dans son film ressemblait donc à une plongée au sein de sa psyché, dans un monde auto-référentiel, où chaque élément trouvait finalement sa réponse et sa raison d'être en lui-même. Il n'a donc jamais été question d'illusion diégétique ${ }^{3}$ dans ses films, de présentation «objective» de la vie, mais toujours d'un regard autoritaire (le terme n'est pas ici à lire comme excluant de ses œuvres une certaine légèreté que l'on retrouve fréquemment) vantant son artificialité.

Avec ses deux derniers films, en choisissant comme sujet l'Histoire, Tarantino a marqué un pas décisif dans son évolution en tant quartiste: son univers n'est plus fermé, il n'est plus tout à fait le seul à pouvoir en changer les règles et, même si nombre de ses thèmes favoris continuent à l'intéresser et à disposer d'une place dans ses œuvres, il fait dialoguer entre elles plusieurs époques, plusieurs modes de pensées, plusieurs temps. Tout en conservant l'ironie, le second degré et la distanciation qui lui sont propres, le regard du spectateur est désormais partie intégrante du discours, ce qui lui assure de ne plus être maintenu artificiellement dans une position d'observateur. Certes Tarantino rejoue l'histoire, la modifie, module son point de vue et les modalités de représentation qu'il emploie, mais il ne peut plus ignorer le poids de son public maintenant qu'il s'intéresse à des récits collectifs.

Son désir de toujours inventer des formes nouvelles ne s'est donc pas tari, si ce n'est qu'il s'exprime désormais avec plus de subtilité : le cinéaste opte dans Django pour une forme de récit relativement éprouvé (un schéma apparemment linéaire, respectant le principe de causalité à la base du classicisme hollywoodien), mais c'est en forçant la mise à distance, en jouant sur une pluralité de points de vue et de niveaux de lecture qu'il balaie l'exigence réaliste qu'aurait pu susciter son projet et dans lequel bon nombre d'auteurs se seraient épanchés. Ce sont plusieurs strates temporelles qui communiquent implicitement par l'intermédiaire du spectateur. Ces dernières n'étant pas physiquement présentes dans le film, le public ne peut que les imaginer à partir des traces laissées par le réalisateur, qui sont autant de légères fissures émaillant le tissu diégétique : chacune d'entre elles est un passage par lequel le regard du spectateur se glisse et questionne le monde qui lui est soumis, laissant ainsi entrer en résonnance plusieurs époques.

Par exemple, lors de la scène de mise à mort de l'esclave D’Artagnan, un jeu de regard confronte le spectateur a sa propre subjectivité : regard froid et sûr de Calvin Candy, symbolisant parfaitement le mode de pensée du bourgeois américain du XIX siècle, persuadé d'être du «bon côté de l'histoire» (comme le montrera le discours du personnage un peu plus tard qui viendra appuyer ses idées d'arguments scientifiques et rationnels afin d'apparaître comme progressiste), regard méprisant et attristé de Django qui nous renvoie à notre position de spectateur actuel (comme nous le verrons

(3) La diégèse est le monde fictionnel de l'œuvre. Chez Tarantino, il n'y a jamais eu le souci de construire un monde que l'on puisse supposer réel (principe de l'illusion diégétique), mais toujours de fantasmer une réalité, de la styliser afin de la transformer. 
ci-dessous), regard outré du Dr Schultz qui image ici les divergences entre la sensibilité européenne, plus policée, et la brutalité des mœurs sudistes.

Le spectateur n'est déjà plus là dans la fiction, mais se trouve face à différents systèmes de pensées appartenant à des temps et des espaces différents, il ne tente donc pas de comprendre l'état d'esprit de l'époque représenté, mais ne s'en détache pas totalement, notamment grâce au personnage du Dr Schultz qui peut être vu comme une métaphore du «sympathique» européen, cultivé et humaniste.

Envisageons maintenant plus en détail les procédés représentationnels que met en œuvre Tarantino pour atteindre ces effets.

Le film joue sur l'artifice et l'exagération dans ses représentations : souffrance des esclaves, sadisme des blancs, mise en scène des personnages. Deux modalités opposées s'affrontent en particulier, l'une sérieuse, l'autre portée sur la dérision : Calvin Candy, propriétaire de Candyland, se met ainsi en scène comme un homme élégant, admirateur de la langue française et préférant être appelé « Monsieur Candy ». Apparaît alors un dispositif théâtral qui trouve sa finalité au moment du dîner (la disposition méticuleuse des couverts, présentée au cours d'une séquence en musique presque religieuse) : les personnages peuvent entrer et sortir de la scène par l'une des nombreuses portes de la pièce, et ainsi pénétrer dans les coulisses (la cuisine, la bibliothèque) où se joue véritablement le drame.

Le spectacle est un des ressorts essentiels de la puissance de Calvin Candy : combat de mandingos, exécutions sauvages. C'est à travers la violence qu'il impose aux autres que se reflète son propre pouvoir.

Le sérieux decepersonnagele metnécessairementàl'écartdu publiccontemporain qui ne peut s'identifier à lui ni sur un mode humoristique (puisque la mise en scène de ce protagoniste exclue justement le registre comique), ni sur un mode réaliste ou historique (puisque, comme nous l'avons vu, cette visée n'est en aucun cas l'objectif affiché par Tarantino).

Django, au contraire, est constamment dans la dérision, déguisé en valet ou en marchand d'esclaves, et semble déjà extérieur à son temps. N'étant pas ancré dans la situation, il peut en jouer comme il le souhaite : il n'hésite pas, par exemple, à imiter la «méchanceté» de Candy quand cela lui semble nécessaire.

Cet aspectle place dans une relation de complicité avec le public:les accoutrements qu'il emploie, les musiques qui lui sont associées (chanson de rap par exemple) lui confèrent une distance vis-à-vis de la situation fictionnelle. C'est donc par le personnage de Django (et dans une moindre mesure du Dr Schultz) que passe notre regard de spectateur contemporain.

Tarantino réussit donc à donner la plus grande légitimité à des personnages comiques (qui comme Django sont finalement les vecteurs de notre regard) et ridiculiser le sérieux des autres, engoncés dans leurs certitudes et incapables de voir l'ironie de la situation et du cadre diégétique (d'autant que l'esclavage sera aboli seulement quelques années après les événements du film).

C'est dans cette opposition que se joue le discours mémoriel mis en place par Tarantino. Les multiples points de vue qui s'expriment tout au long du film nous 
offrent un panorama de la situation. Certaines voix sont largement occultées : la représentation sérieuse de Candy le place dans l'Histoire et l'accable du poids de ses actes, il est comme le pantin duquel s'amusent les spectateurs d'aujourd'hui, complices de Django, regardant celui qui s'agite et légitime un ordre qui n’a plus de sens. Il est le personnage sérieux à l'intérieur d'un système porté entièrement par la dérision, ce qui le rend ridicule et absurde et décrédibilise de la manière la plus efficace qui soit toute tentative de pensée chez les esclavagistes. Mais, l'identification complaisante au personnage de Django, cet esclave élevé en porte-parole du spectateur actuel en quête de justice, venu balayer les abus de l'époque, est également symptomatique de la simplification du travail mémoriel à l'œuvre chez certains (la destruction du domaine à la fin du film symbolise clairement cette idée de faire table rase du passé, mais limite par là même la possibilité d'une véritable réflexion sur l'esclavage). Le cinéaste condamne en quelque sorte ce désir contemporain de détruire le passé pour le réduire à quelques formules lapidaires, témoignant par là d'un monde qui, s'il a vite fait de condamner les injustices, ne s'intéresse pas à les comprendre et à les expliquer, se soucie du plus superficiel là où l'étude approfondie pourrait l'éclairer sur son état actuel.

L'idée de faire communiquer de la sorte différentes temporalités est donc un moyen pour Tarantino d'exprimer la difficulté rencontrée quand il s'agit de réfléchir à des sujets complexes et toujours tabous comme celui-ci.

La mise à distance est le procédé cinématographique moderne par excellence devenu nécessaire pour le travail mémoriel : les cinéastes ont inventé de multiples dispositifs leur permettant d'éviter l'immersion trop brutale du spectateur qui réduirait toute tentative de pensée à néant.

Dans ces films, ce souci répond également à une question d'énonciation, il s'agit d'affirmer un point de vue, une façon particulière de regarder la situation, de la représenter. La mise en scène n'est plus une illustration (même si en vérité elle ne l'a jamais vraiment été), mais un acte ayant conscience de sa portée.

Cette conception se résume parfaitement dans l'article De l'abjection de Jacques Rivette. En condamnant aussi fermement le cinéaste italien Gilles Pontecorvo, Rivette marque les bases de ce que l'on pourrait appeler une morale de la représentation : un questionnement nécessaire de la part du cinéaste qui souhaiterait se pencher sur des sujets éthiquement complexes, notamment à propos du réalisme et de la morale (fautil montrer tel élément ou simplement le suggérer ? Faut-il traiter telle thématique de façon mimétique ou détournée?).

Cette morale cinématographique suggère certes un regard distancié vis-à-vis de la situation fictionnelle, mais nécessite tout de même un véritable monde diégétique. Le spectateur peut en sortir à certains moments et juger le film, sa construction et son organisation, néanmoins il se reportera toujours à lui.

Si Tarantino se moque de ces considérations et n'hésite pas à figurer frontalement l'horreur, c'est tout simplement que chez lui l'univers fictionnel n'a pas d'existence réelle: sa porosité est telle que le public n'est jamais intégré à un récit, un nombre incalculable d'éléments disparates l'entraînant dans des directions diverses au 
sein desquelles le monde décrit n'est plus qu'un référent lointain (d'autres auteurs «modernes» ont pu arriver à de telles extrémités, comme Godard par exemple ou Wim Wenders, mais sans traiter de thèmes mémoriels, ce qui les conduisait à avoir une toute autre relation à la morale). Chez le cinéaste, montrer un esclave se faire dévorer par des chiens ne renvoie pas à un réel précis, et ne peut donc être taxé d’immoralité.

Spielberg, lui, cherche l'immersion du spectateur : cependant, il évite de figurer l'esclavage et se concentre sur une mémoire du politique. Par là, il évite l'aspect dérangeant de la question morale et, en se concentrant sur la figure de Lincoln, donne à son film des couleurs utopiques. Or l'utopie est, par définition, étrangère à ces problèmes éthiques, car elle suppose que ceux-ci sont déjà résolus. Comme nous l'avons vu, la pureté de la figure de Lincoln rachète la moralité douteuse que peut proposer la démocratie, rendant ainsi libre le cinéaste de ses choix de représentations.

Les deux réalisateurs, en empruntant des voies extrêmement diverses, réussissent donc à passer outre le dogme moraliste qui prévaut au cinéma depuis les années 1960. 